\title{
Genetic variation associated with increased insecticide resistance in the malaria mosquito, Anopheles coluzzii
}

\author{
Bradley J. Main ${ }^{1 *}$, Amanda Everitt', Anthony J. Cornel ${ }^{2}$, Fereydoun Hormozdiari ${ }^{3}$ and Gregory C. Lanzaro ${ }^{1}$
}

\begin{abstract}
Background: Malaria mortality rates in sub-Saharan Africa have declined significantly in recent years as a result of increased insecticide-treated bed net (ITN) usage. A major challenge to further progress is the emergence and spread of insecticide resistance alleles in the Anopheles mosquito vectors, like An. coluzzii. A non-synonymous mutation in the para voltage-gated sodium channel gene reduces pyrethroid-binding affinity, resulting in knockdown resistance $(k d r)$. Metabolic mechanisms of insecticide resistance involving detoxification genes like cytochrome P450 genes, carboxylesterases, and glutathione S-transferases are also important. As some gene activity is tissue-specific and/or environmentally induced, gene regulatory variation may be overlooked when comparing expression from whole mosquito bodies under standard rearing conditions.

Results: We detected complex insecticide resistance in a 2014 An. coluzzii colony from southern Mali using bottle bioassays. Additional bioassays involving recombinant genotypes from a cross with a relatively susceptible 1995 An. coluzzii colony from Mali confirmed the importance of $k d r$ and associated increased permethrin resistance to the CYP9K1 locus on the $X$ chromosome. Significant differential expression of CYP9K1 was not observed among these colonies in Malpighian tubules. However, the P450 gene CYP6Z1 was overexpressed in resistant individuals following sublethal permethrin exposure and the carboxylesterase gene COEAE5G was constitutively overexpressed.

Conclusions: The significant P450-related insecticide resistance observed in the 2014 An. coluzzii colony indicates that ITNs treated with the P450 inhibitor piperonyl butoxide (PBO) would be more effective in this region. The known insecticide resistance gene CYP6Z1 was differentially expressed exclusively in the context of sublethal permethrin exposure, highlighting the importance of tissue-specificity and environmental conditions in gene expression studies. The increased activity of the carboxylesterase COEAE5G in the resistant An. coluzzii colony suggests resistance to other insecticides like organophosphates. Additional gene expression studies involving other tissues (e.g. fat body) would provide a more comprehensive view of genes underlying metabolic insecticide resistance in An. coluzzii from Mali. Identifying genetic markers linked to these regulatory alleles is an important next step that would substantially improve insecticide resistance surveillance and population genetic studies in this important vector species.
\end{abstract}

Keywords: Anopheles, Malaria vector, Insecticide resistance, Adaptive introgression, kdr, P450, Carboxylesterase

\footnotetext{
* Correspondence: bradmain@gmail.com

'Department of Pathology, Microbiology, and Immunology, UC Davis, Davis,

CA 95616, USA

Full list of author information is available at the end of the article
} 


\section{Background}

Malaria deaths have been cut in half since 2000, due in large part to insecticide-treated bed net (ITN) campaigns and use in sub-Saharan African countries like Mali [1]. A major threat to further reductions in malaria incidents is the emergence and spread of insecticide resistance (IR) in Anopheles (An.) mosquitoes like An. gambiae and $A n$. coluzzii: the primary mosquito vectors of malaria in Mali [2-4]. Anopheles coluzzii has been increasing in relative abundance coincident with the adaptive introgression of the knockdown resistance $(k d r)$ allele from An. gambiae in 2006 and is now the major malaria vector in southern Mali [4-6]. $K d r$ refers to nonsynonymous mutations in the voltage-gated sodium channel gene (para), which reduces pyrethroid-binding affinity; an insecticide resistance mechanism called target site resistance. The $k d r$ allele has been increasing in geographical distribution and relative frequency throughout Africa, apparently in response to increased ITN use [7-9]. Detoxification genes like cytochrome P450 genes, carboxylesterases, and glutathione S-transferases (GSTs) can also confer resistance to insecticides [10-14]. For example, P450 genes like CYP9K1 [15, 16], CYP6P3 [12], CYP6M2 [13], CYP6Z1 [17], CYP325A3 [18, 19], and others [20, 21] have been associated with insecticide resistance in anophelines. Insecticide resistance ultimately is the combination of multiple possible mechanisms including target site and metabolic resistance, as well as reduced cuticle penetrance $[22,23]$. P450 genes and $k d r$ may also act synergistically [24].

Genomic studies commonly focus on single nucleotide polymorphisms because they can be readily identified with short reads. However, there is evidence that structural variants, including inversions, duplications, and deletions may be involved in adaptation and speciation within the Anopheles gambiae complex. For example, distinct inversion states and microsatellite profiles on chromosome 2 have been associated with reproductively isolated populations of $A n$. gambiae, previously identified as the Bamako, Savanna, and Mopti (i.e. An. coluzzii) chromosomal forms [25]. In Drosophila, P450 gene duplication has been linked to DDT resistance [26]. Thus, in this study, we examine structural and regulatory variation in an $A n$. coluzzii population in southern Mali that is associated with increased insecticide resistance and a previously described selective sweep [4]. Due to the importance of tissue-specificity in gene expression assays [27] and active expression of the candidate P450 gene CYP9K1 in Malpighian tubules [28], we used RNAseq to examine expression differences between alternative haplotypes at CYP9K1 (cyp-1 and cyp-2) in Malpighian tubules. We also compared expression profiles after sublethal exposure to permethrin, as some genes in $A n$. gambiae may be responsive to permethrin [29].

\section{Methods \\ Mosquitoes}

We assessed insecticide resistance in the following mosquito strains from Mali: 1995 An. coluzzii (MOPTI; MRA-763), 2014 An. coluzzii (Selenkenyi), and $A n$. gambiae (pimperena; MRA-861).

\section{Insecticide resistance bioassays}

To test if metabolic insecticide resistance (P450-related) and overall insecticide resistance has increased in the modern An. coluzzii population in southern Mali since the start of a major insecticide-treated bed net campaign in 2006, we performed insecticide resistance bottle bioassays, following Centers for Disease Control and World Health Organization guidelines [30,31] on the 2014 and 1995 An. coluzzii colonies (and An. gambiae pimperena for reference). We tested the effects of permethrin alone and permethrin immediately after exposure to the P450 inhibitor piperonyl butoxide (PBO). In brief, mosquitoes were placed in a $250 \mathrm{ml}$ Wheaton bottle coated with $400 \mu \mathrm{g}$ of PBO (in an acetone solution) for $1 \mathrm{~h}$. Then, the mosquitoes were transferred to another $250 \mathrm{ml}$ Wheaton bottle coated with $21.5 \mu \mathrm{g}$ of permethrin and knockdown mosquitoes were recorded every $15 \min$ for $1 \mathrm{~h}$. After these incubation periods, all living mosquitoes were placed in a pint-sized paper cage with constant access to $10 \%$ sugar solution. Then, mortality was assessed after $24 \mathrm{~h}$. To estimate the effects of permethrin alone, the PBO treatment bottle was replaced with a control bottle coated with pure acetone. Deleterious effects from the experimental procedure of transferring mosquitoes between Wheaton bottles was assessed by replacing both the PBO- and permethrin-treated bottles with bottles treated with pure acetone.

\section{Individual mosquito bottle bioassays with F2 recombinant genotypes}

We generated F2 mosquitoes by crossing $1995 A n$. coluzzii (cyp-2 genotype at CYP9K1) virgin females with 2014 An. coluzzii (cyp-1 genotype at CYP9K1) males to make $\mathrm{F} 1$ hybrids and then the $\mathrm{F} 1$ generation was allowed to interbreed. As CYP9K1 is on the $\mathrm{X}$ chromosome, all F1 males were hemizygous cyp-2. In the F2 generation, female genotypes were expected to be a 50:50 mix of cyp-1/2 and cyp-2/2. F2 male genotypes were expected to be a $50: 50$ mix of hemizygous $c y p-1$ or $c y p-2$. The permethrin resistance bioassays were conducted using $250 \mathrm{ml}$ Wheaton bottles that were prepared as previously described. Between 10 am and 3pm, we placed each individual mosquito in a permethrin-treated bottle for at least $1 \mathrm{~h}$ and recorded when the individual was not able to stay upright or maintain normal flight (knockdown) upon disturbing the bottle and rotating it 360 degrees in the horizontal position. Immediately 
following knockdown, each individual was placed in a 1.5 $\mathrm{ml}$ tube and snap frozen in an aluminum block in dry ice and stored at $-80^{\circ} \mathrm{C}$ until DNA extraction.

\section{Transcriptome analysis following sublethal permethrin exposure}

To induce expression of genes that may be responsive to permethrin [29], 3-day-old virgin females were exposed to a sublethal dose of permethrin $(1 / 4 \times$ standard dose $21.5 \mu \mathrm{g} / \mathrm{l})$ in a Wheaton bottle for $15 \mathrm{~min}$. Control mosquitoes were exposed to acetone-treated bottles. Then mosquitoes were given a $4 \mathrm{~h}$ recovery period in a pint cage with constant access to a $10 \%$ sugar solution. This recovery time was chosen based on previous gene expression results following permethrin exposure in An. gambiae [29]. Mosquitoes were cold-anesthetized by placing the cage in a $-20{ }^{\circ} \mathrm{C}$ freezer for $3 \mathrm{~min}$ immediately prior to dissections.

\section{Malpighian tubule dissection and RNA isolation}

All dissections were performed between 1-4 pm to limit gene expression differences due to circadian rhythm genes. To isolate the Malpighian tubules, the penultimate abdominal segment of a cold-anesthetized female was gently pulled with a micro-tweezer until the gut, Malpighian tubules, and ovaries were isolated from the carcass. Following removal of the ovaries and foregut, the Malpighian tubules (and some hindgut) were then immediately transferred into lysis buffer (Zymo Research, Irvine, USA) and homogenized with a micropestle (Additional file 1: Table S1). Total RNA (and DNA in parallel) was then extracted from individual females or pools of 8-12 Malpighian tubules per biological replicate using the ZRDuet kit (Zymo Research) with an on-column DNAse treatment step.

\section{Transcriptome sequencing and QC}

We generated strand-specific mRNA-seq libraries (KAPA Biosystems, Wilmington, USA) for 6 biological replicates of control and treatment conditions for both the $1995 \mathrm{An}$. coluzzii (cyp-2) and 2014 An. coluzzii (cyp-1) genotypes (Additional file: Table S2). These 24 libraries were sequenced on a single lane of HiSeq3000 SR50 at the UC Davis DNA Technologies Core. Poor quality bases and Illumina adapter sequences were trimmed from the raw reads using Trimmomatic version 0.30 [32], with the following parameters: LEADING:3, TRAILING:3, SLIDINGWINDOW:4:15, MINLEN:36. The trimmed reads were then assessed for general quality using FastQC version 0.10.1 (Babraham Bioinformatics, Cambridge, UK).

Read mapping and differential gene expression analysis Single-end reads were mapped with STAR version 2.5 (-quantMode GeneCounts; [33]) to the An. coluzzii reference genome (scaffolds AcolM1) with gene annotation information (AcolM1.3.gtf; Additional file 1: Table S2). A detailed workflow of our differential expression analysis is available as a Jupyter notebook (Additional file 1: Table S3). In brief, gene level counts were converted to counts per million (cpm) using edgeR [34] and we removed genes with very low expression levels by requiring at least 5 libraries with a cpm $>2$ for each gene. After this filtering step, we were left with 8485 genes that we tested for differential expression. We further normalized individual samples using the trimmed mean of M-values (TMM) method. To estimate differential expression between genotypes and between treatments, we used voom [35] in the limma package [36] to perform a variancestabilizing transformation on the raw counts and then fit a linear model using lmFit [37]. We applied a cell means model including genotype and treatment after accounting for potential batch effects at the dissection stage. To identify differentially expressed (DE) genes we used the eBayes function [35] and BenjaminiHochberg (FDR)-corrected $P$-values $(<0.05)$ from the decideTests function.

We classified a gene as a putative detoxification gene if it was included in the detoxification chip [18], which includes over 200 genes belonging to at least three major enzyme families: glutathione S-transferases, cytochrome P450 monooxygenases and esterases.

\section{Analyzing structural variation associated with post-2006 An. coluzzii}

Structural variants were detected in whole genome sequencing data (Additional file 1: Table S4) using LUMPY version 0.2.13 [38]. Discordant paired-end and split-end reads were extracted using samtools. Library size, mean, and standard deviation were estimated using paired_distro.py script from LUMPY. The inferred breakpoints produced by LUMPY were genotyped using SVtyper version 0.1.2. Calls identified as break ends were removed and analyzed separately. Remaining inferred breakpoints were used if they were larger than $100 \mathrm{bp}$ in size, were not homozygous reference in all samples, and had more than ten supporting reads in the two highest coverage samples. For remaining calls, the ratio of cyp-1 samples having either a heterozygous or homozygous alternate to the total number of cyp-1 samples possible was calculated. This was repeated for cyp-2 samples, and Fisher's exact test was performed to determine which SVs differed in frequency between the two populations.

Inferred breakpoints which corresponded to potential repeat elements and genic regions were determined using bedtools intersect version 2.17.0. For repeat elements, a $50 \%$ reciprocal overlap between structural variant call and repeat elements was required. For genic regions, one breakpoint must have occurred within $500 \mathrm{bp}$ of gene. 


\section{Results}

Testing for increased permethrin resistance in An. coluzzii from southern Mali

According to the World Health Organization, a mosquito colony is considered resistant if less than $90 \%$ mortality is observed at $24 \mathrm{~h}$ post-exposure and susceptible if mortality is greater than 98\% [31]. The 2014 An. coluzzii and An. gambiae colonies were both considered resistant, with $68 \%$ and $57 \%$ mortality after $24 \mathrm{~h}$, respectively. The 1995 An. coluzzii colony was relatively susceptible with a mortality rate of $95 \% 24 \mathrm{~h}$ postexposure to permethrin (Fig. 1a). To compare the knockdown phenotype in response to permethrin between colonies we estimated the knockdown times for $50 \%(\mathrm{kdT} 50)$ of mosquitoes in each bottle replicate. The mean kdT50 for 2014 An. coluzzii was 29 min, which was significantly longer (more resistant) than the 1995 An. coluzzii colony (7.5 min, t-test, $\left.t_{(6)}=2.9, P=0.02\right)$. Estimated knockdown times with permethrin exposure were not significantly different between 2014 An. coluz$z i i$ (29 $\mathrm{min}$ ) and An. gambiae (35 min, t-test, $t_{(6)}=0.66$, $P=0.54$; Fig. 1b).

To estimate permethrin resistance due to P450 activity, we compared mortality rates and knockdown times with and without pre-treatment of CDC bottles with the general P450 inhibitor piperonyl butoxide (PBO). Mortality significantly increased in the PBO-treated group of 2014 An. coluzzii from $67 \%(n=43)$ to $98 \%(n=53$, Fisher's exact test, $P<0.0001$; Fig. 1a). Pre-treatment with PBO reduced the kdT50 for 2014 An. coluzzii from 28.75 to 21.25 min, but this change was not significant (t-test, $t_{(6)}=1.03, P$ $=0.34$ ). Similar trends were observed in An. gambiae, but no significant PBO-effect was observed for mortality or kdT50 in the 1995 An. coluzzii colony (Fig. 1).

\section{Increased resistance in An. coluzzii is associated with a selective sweep on the $X$ chromosome}

A previous study identified multiple $\sim 150 \mathrm{~kb}$ haplotypes at $\sim 15 \mathrm{Mb}$ on the $\mathrm{X}$ chromosome, namely cyp-1, cyp -2 , and cyp-3 [4]. After 2006, cyp-1 became fixed in the population, resulting in a selective sweep. To test if genetic variation associated with the cyp-1 haplotype confers insecticide resistance, we performed single-mosquito permethrin bottle bioassays on 122 female and 141 male F2 genotypes from a cross between cyp-2 females (1995 An. coluzzii) and cyp-1 males (2014 An. coluzzii). After the bioassay was completed, each mosquito was genotyped for cyp haplotype and $k d r$. Due to the direction of the initial cross, homozygous cyp-1 genotypes were not generated. Individuals that survived beyond $60 \mathrm{~min}$ were considered resistant. There was a significant $k d r$ effect on the proportion resistant in both males and females $\left(\chi^{2}=24.99, d f=1, P<0.0001\right.$, Fig. 2$)$. In females, the cyp-1 haplotype was associated with higher resistance $\left(\chi^{2}=3.915, d f=1, P=0.047\right.$, Fig. 2$)$. This cyp- 1 effect was not observed in males.

\section{Genome structural variation in An. coluzzii}

To investigate whether structural variation was selected for in An. coluzzii in addition to the cyp-1 haplotype and $k d r$, we used the program LUMPY to call inferred breakpoints (edges of structural variants) genome-wide. We utilized 29 An. coluzzii genomes, including 17 cyp-1 and 12 cyp-2 individuals (Additional file 1: Table S4). After filtering, we identified 12,255 structural variants (SVs), including 9617 deletions, 1731 duplications, and 907 inversions (Additional file 1: Table S5). Of the SVs, 5739 overlapped with a repeat element and 5072 occurred within 500 bp of a known gene. A total of 66 SVs involve the $c y p-1$ selective sweep region on the $\mathrm{X}$ chromosome. Most of these SVs (50/66) completely overlap the cyp-1 haplotype region $(\sim 150 \mathrm{~kb})$, while $6 \mathrm{SVs}$ occur completely within the cyp-1 haplotype region. A total of 223 SVs differed between the pre-2006 cyp-2 and post-2006 cyp-1 populations, using Fisher's exact test. The 223 SV genes overlap 10,968 genes. However, if we exclude large (> $10 \mathrm{~kb})$ SVs, 137 genes remained at or near $(<500 \mathrm{bp})$ SVs and the coding regions of 35 genes were overlapped by at least $1 \mathrm{bp}$ (Additional file 1: Table S6). We did not identify known or putative insecticide resistance genes in these regions. To test for enrichment of these 137 genes in gene functional category, we performed a gene ontology analysis using g:profiler [39]. This is important because it is possible that the contribution of mutations involving several genes in the same biological pathway result in an overall adaptive phenotype (e.g. insecticide resistance). Enrichment was observed in the "regulation of Ras protein signal transduction" term (biological process) and three molecular functions: Rho guanyl-nucleotide exchange factor activity, protein binding, and molecular function regulator (Additional file 1: Table S7).

\section{Malpighian tubule-specific differential expression analysis} To elucidate genes underlying the increased insecticide resistance of the 2014 An. coluzzii colony, we compared gene expression profiles with the relatively susceptible 1995 An. coluzzii colony under control and sublethal permethrin exposure conditions. We generated 6 biological replicates of strand-specific mRNA-seq libraries from female Malpighian tubule tissue (see Methods) for each genotype and condition. The RNA-seq libraries were sequenced with single-end reads with a median of 15.5 million reads (Additional file 1: Table S2). Candidate genes that underlie increased insecticide resistance in the 2014 An. coluzzii colony were identified based on the assumption that resistance genes will be enriched among the total differentially expressed genes detected between colonies. 

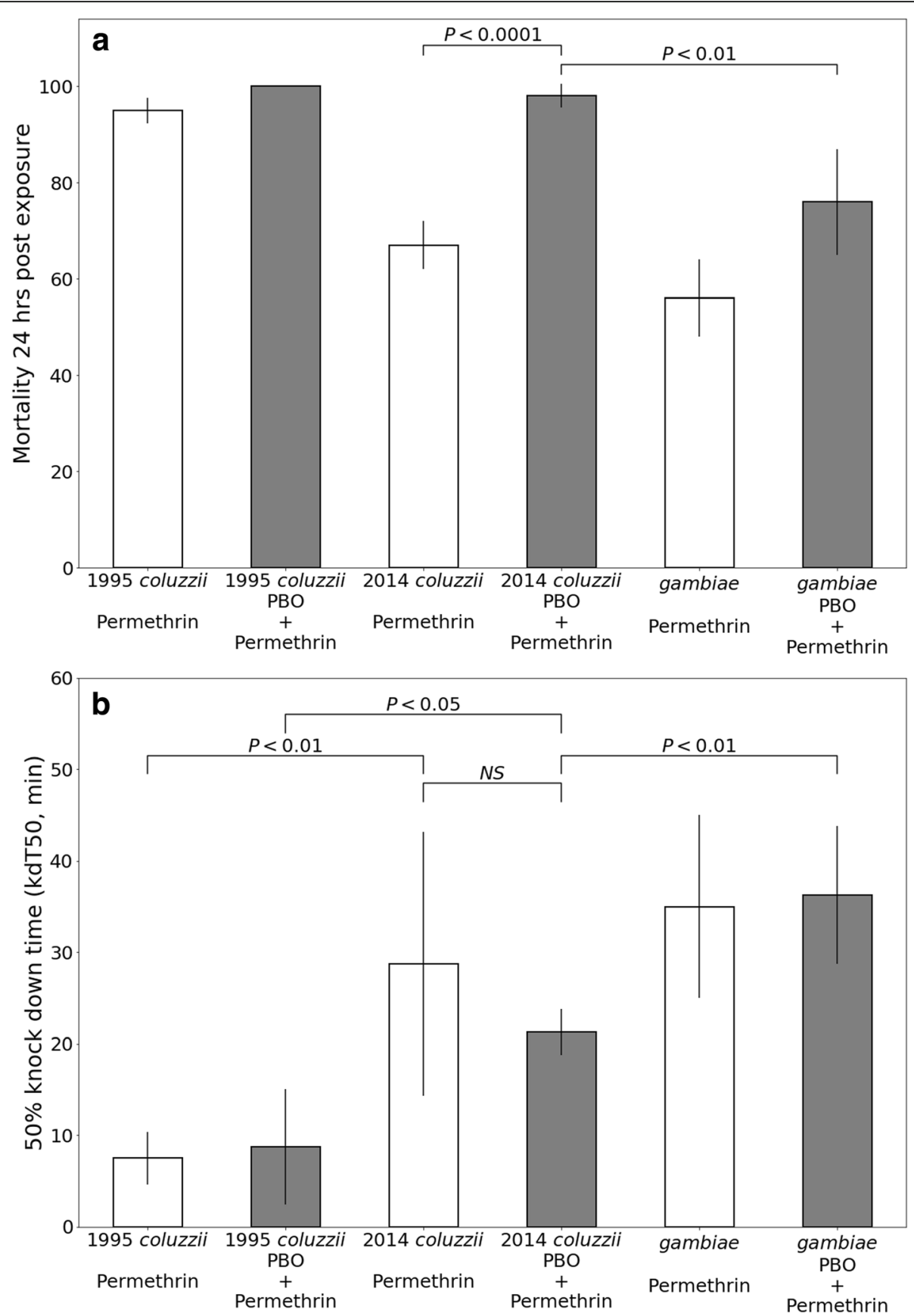

Fig. 1 Estimating permethrin resistance related to P450 activity. a Mortality rates following permethrin exposure (white bars) and PBO + permethrin exposure (grey bars). The Anopheles genotype tested is indicated on the X-axis. Error bars indicate standard error. $P$-values were calculated using Fisher's exact test. b Knockdown times following permethrin exposure (white bars) and PBO + permethrin exposure (grey bars). The Y-axis is the time (minutes) until 50\% of the individuals were considered knocked down (kdT50). Each assay was performed with 4 replicate cages of 10 individuals each. Error bars indicate the 95\% confidence interval. P-values were calculated using a t-test

A total of 8485 genes were tested for differential expression after requiring that a given gene was expressed above 2 counts per million in at least 5 biological replicates (Additional file 1: Table S8). Twenty-two differentially expressed genes between the colonies were unique to the treatment groups (Fig. 3a, b-3), 65 were unique to the control group comparison (Fig. 3a, b-4), and 61 were differentially expressed between the colonies under both treatment (Fig. 3b-3) and control conditions (Fig. 3a, b-4; Additional file 1: Table S9). We did not detect significant differentially expressed genes between control and sublethal permethrin treatment groups of either colony (Fig. 3b-1 and b-2). 


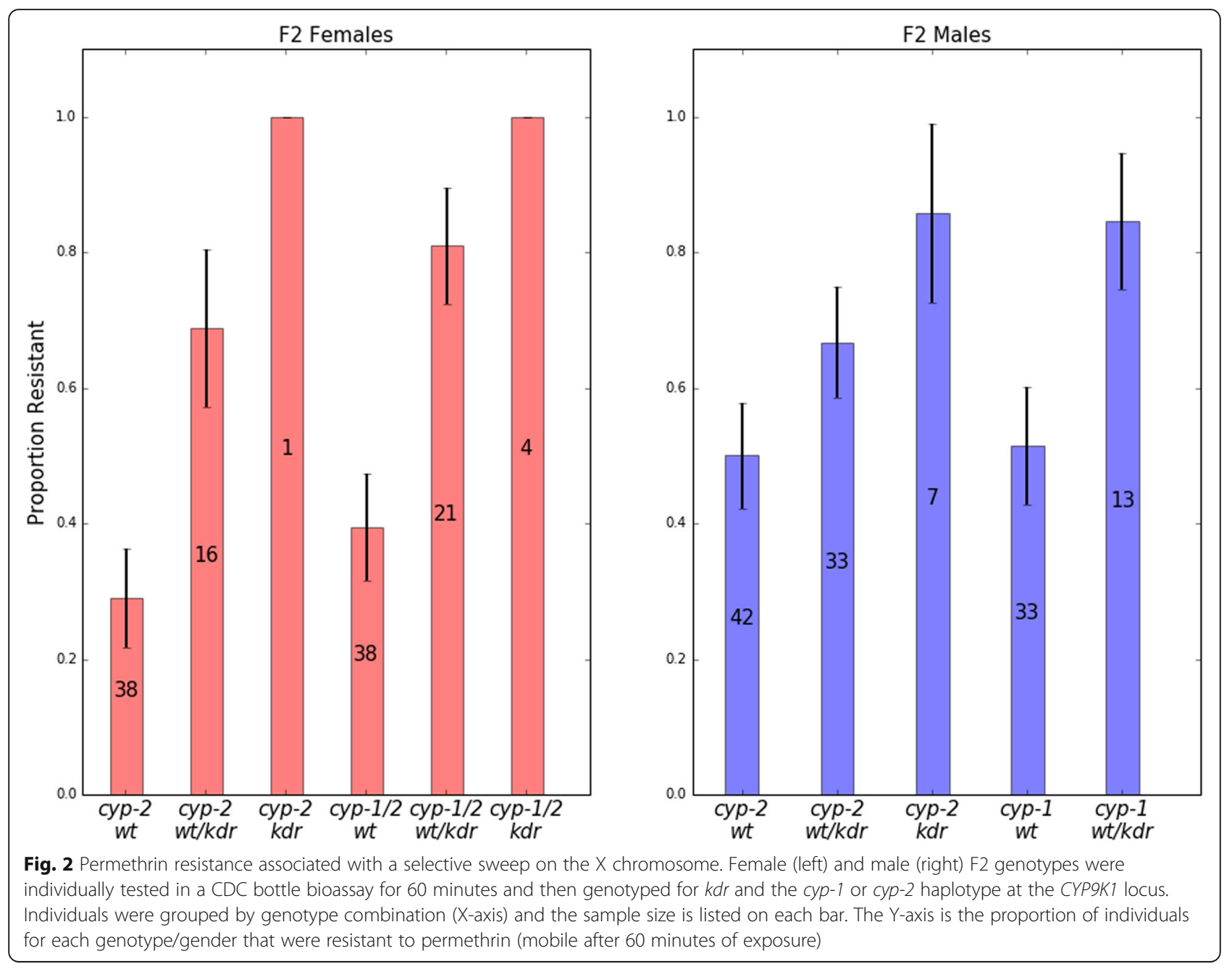

The P450 gene CYP9K1 was not differentially expressed between the colonies but tended toward under-expression in the 2014 An. coluzzii colony. Under control conditions the log fold change (FC) was $-0.56(P=0.32)$ and under treatment conditions the $\operatorname{logFC}$ was $-0.04(P=0.96)$,

indicative of underexpression of the cyp-1 allele (2014 An. coluzzii) compared to the cyp-2 allele (1995 An. coluzzii). We also detected under-expression of the cyp-1 allele in individual female F1 hybrid Malpighian tubules under control [Log2 (cyp-1/cyp-2) $=-0.74]$ and
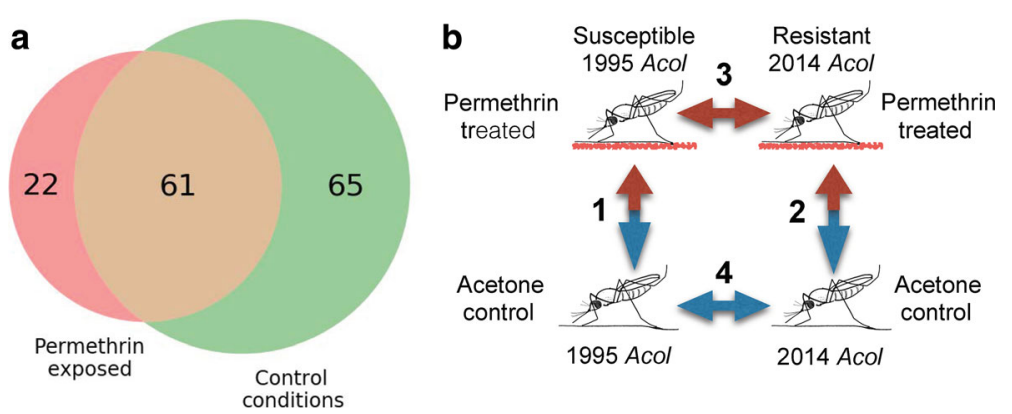

Fig. 3 Differential expression analysis. a Numbers of differentially expressed genes in An. coluzzii Malpighian tubules associated with permethrin resistance following sublethal permethrin exposure and control conditions. Sixty-one genes are DE in both conditions. b We tested for differentially expressed genes between control and treatment conditions (1 and 2) and between An. coluzzii strains following sublethal permethrin exposure (3) and under control conditions (4) 
permethrin-treated conditions [Log2 (cyp-1/cyp-2) $=-0.84$ ] (Additional file 1: Table S10).

We performed a gene functional enrichment analysis of all differentially expressed genes between the colonies, including control and treatment conditions using g:profiler [39]. This test resulted in an enrichment of genes in the steroid biosynthesis pathway (KEGG:00100) and GO terms related to several metabolic processes, glucuronidation, and flavonoid biosynthesis (Additional file 1: Table S11). There was no overlap between polymorphic structural variants (i.e. indels $<10 \mathrm{~kb})$ and the differentially expressed genes detected in this study.

\section{Detoxification genes}

Three of the DE genes between the 2014 An. coluzzii cyp-1 colony and the 1995 An. coluzzii cyp-2 colony under treatment conditions and three DE genes under control conditions were known detoxification genes. The carboxylesterase gene COEAE5G was upregulated in the resistant 2014 An. coluzzii cyp-1 colony in both permethrin-treated $(\operatorname{logFC} 1.68$, adj. $P<0.001)$ and control conditions $(\operatorname{logFC} 1.07$, adj. $P<0.01)$. The $\mathrm{P} 450$ gene CYP6Z1 was upregulated in the resistant 2014 An. coluzzii cyp-1 colony (logFC 0.94, adj. $P<0.05$ ) and the glutathione S-transferase (GSTu2) was downregulated in the 2014 An. coluzzii cyp- 1 individuals (logFC -1.39 , adj. $P<0.05$ ) in the context of sublethal permethrin exposure. Detoxification genes that were DE in the control conditions included the P450 gene CYP4H19 $(\operatorname{logFC}-2.04$, adj. $P<0$. $01)$ and the thioredoxin peroxidase 4 (TPX4, $\log \mathrm{FC}-2.05$, adj. $P<0.05)$.

\section{Discussion}

Using bottle bioassays we demonstrate that the 2014 An. coluzzii colony was more resistant than the 1995 An. coluzzii colony in terms of knockdown time and mortality at 24 hours. PBO assays indicated that P450 genes confer added insecticide resistance to the 2014 An. coluzzii colony. High susceptibility in the 1995 An. coluzzii colony inhibited our ability to estimate the contribution of $\mathrm{P} 450$ genes to resistance in this colony.

Previous studies have identified a selective sweep on the $\mathrm{X}$ chromosome of An. coluzzii associated with increased relative fitness [4]. CDC bottle bioassays on individual F2 recombinant individuals confirm the known role of $k d r$ in permethrin resistance and support a link between the selected haplotype (cyp-1) and increased resistance.

This is the first study to examine structural variation within An. coluzzii populations. This is important because it is known that insects can gain insecticide resistance via copy number variation [26].
The SVs that were polymorphic between pre- and post-2006 An. coluzzii occurred at or near 137 genes. None of these genes are known detoxification genes [18]. At least one SV occurred in the intron of $k d r$, potentially affecting gene expression levels, but we did not detect differential expression of the para gene.

Among genes that may be affected by the polymorphic SVs, we detected an enrichment of genes in the "regulation of Ras protein signal transduction" biological process and the Rho guanyl-nucleotide exchange factor activity molecular function. Ras proteins are small GTPases that are involved in general signal transduction and cell growth. Rho guanyl-nucleotide exchange factor activity is involved in the exchange of guanyl nucleotides for a Rho GTPase. Further studies are needed to test if and how specific signal transduction genes (i.e. GTPases) are affected by SVs that may be under selection in these An. coluzzii populations.

Multiple studies have explored gene expression differences associated with insecticide resistance by grinding up whole body individuals from a colony that has been identified as resistant or susceptible. However, this approach may overlook genes that are highly tissue-specific [28] or that are responsive to environmental stimuli, like permethrin exposure [29]. As testing multiple tissues was beyond the scope of this study, we focused on expression differences in Malpighian tubules because this tissue is important for detoxification and the candidate $\mathrm{P} 450$ gene CYP9K1 has been shown to be highly expressed in this tissue [28]. This approach increased the sensitivity to detect differences in genes that are active in Malpighian tubules (and attached hindgut), while decreasing our ability to identify DE genes that are more active in other tissues, like the fat body.

The carboxylesterase gene COEAE5G is constitutively overexpressed in the resistant cyp-1 2014 colony compared to the relatively susceptible cyp-2 $1995 \mathrm{An}$. coluz$z i i$ colony and the $\operatorname{logFC}$ increased under sublethal permethrin exposure conditions $(\log \mathrm{FC} 1.07$ vs 1.68). Thus, the COEAE5G allele in the resistant An. coluzzii may also be more responsive to permethrin exposure. Further examination of COEAE5G activity in different resistant An. coluzzii colonies, tissue types and environmental conditions and its association with insecticide resistance is needed to assess the relative importance of COEAE5G in elevated insecticide resistance in Mali.

The P450 gene CYP4H19 was downregulated in the cyp-1 colony under control conditions. While resistance genes are commonly upregulated, P450 gene activity can have toxic effects [40] and downregulation of P450 genes has been previously reported in resistant colonies $[18,41]$. Interestingly, the antioxidant TPX4 was also 
downregulated in the cyp-1 colony by more than twofold under control conditions. Downregulation of TPX4 was also observed in the DDT resistant colony ZAN/U but not the RSP resistant strain [18]. Thus, insecticide resistance involves multiple mechanisms, which may have distinct compensatory strategies, including downregulation of particular genes.

The P450 gene CYP6Z1 has been shown to metabolize permethrin [17, 42-44] and was upregulated in the cyp1 resistant An. coluzzii colony following sublethal permethrin exposure, but not under control conditions. This indicates that genetic variation in the cyp-1 colony increases the responsiveness of CYP6Z1 to permethrin exposure. This is an important finding because candidate insecticide resistance genes like CYP6Z1 can be missed with typical approaches involving whole body mosquitoes under standard rearing conditions. Additional tissue-specific gene expression studies in hybrid individuals would elucidate whether this regulatory variation is at CYP6Z1 (cis) or associated with upstream transcription factors (trans).

\section{Conclusions}

We detected complex insecticide resistance in $A n$. coluzzii from southern Mali, supporting the use of $\mathrm{PBO}$ in bed nets in this region. The $\mathrm{P} 450$ gene CYP6Z1 was overexpressed in Malpighian tubules of resistant individuals following sublethal permethrin exposure. This finding highlights the importance of tissue-specificity and environmental conditions in gene expression studies. The carboxylesterase gene COEAE5G was constitutively overexpressed in resistant $A n$. coluzzii, suggestive of resistance to other insecticides like organophosphates. Bioassays involving recombinant genotypes confirmed the importance of $k d r$ and the cyp-1 haplotype at the CYP9K1 locus in knockdown resistance to permethrin. However, significant differential expression of CYP9K1 was not observed in Malpighian tubules. Additional expression studies involving other tissues (e.g. fat body) would provide a more comprehensive view of genes underlying metabolic insecticide resistance in An. coluzzii from Mali. Identifying genetic markers linked to these regulatory alleles is an important next step that would substantially improve insecticide resistance surveillance and population genetic studies in this important vector species.

\section{Additional file}

Additional file 1: Table S1. This table provides links to video examples of the Malpighian tubule dissections described in the methods. Table S2. Illumina sequencing read depths per mRNAseq library and associated metadata. Table S3. A Jupyter notebook describing the differential expression workflow. Table S4. Genome coverage data. Table S5. Genome-wide structural variant calls in An. coluzzii. Table S6. A list of genes that were overlapped by a structural variant $(<10 \mathrm{~kb})$. Table S7. Gene ontology results from genes in table S5. Table S8. mRNAseq count data for each gene and corresponding An. gambiae ortholog. Table S9. All differentially expressed genes. Table S10. This Jupyter notebook describes the allele-specific expression analysis of CYP9K1 in a сур-1/cyp-2 F1 hybrid. Table S11. Gene ontology results. (XLSX 3557 kb)

\section{Abbreviations}

WHO: World Health Organization; ITN: insecticide-treated bed net; kdr: knockdown resistance; CDC: Center for Disease Control;

kdT50: knockdown time for 50\% of the sample; DE: differential expression;

SV: structural variant; GO: Gene Ontology; PBO: piperonyl butoxide;

$\mathrm{Cl}$ : confidence interval

\section{Acknowledgements}

Thanks to Youki Yamasaki for assistance with mosquito dissections and Katherine Brisco for assistance with bioassays. The sequencing was carried by the DNA Technologies Core and assistance was provided by the Expression Analysis Core at the UC Davis Genome Center, supported by NIH Shared Instrumentation Grant 1S100D010786-01.

\section{Funding}

This study was supported by the National Institute of Allergy and Infectious Diseases (R21Al117174 to BJM).

\section{Availability of data and materials}

The data associated with this manuscript are included in the article and its additional files. Sequencing reads are available on SRA (PRJNA390156).

\section{Authors' contributions}

BJM conceived and designed the study. BJM supervised the study. BJM and AE performed the study. AJC assisted with the bioassays. AJC and GCL collected colony material. FH assisted with structural variant study. BJM and $\mathrm{AE}$ analyzed the data and wrote the manuscript. All authors read and approved the final manuscript.

\section{Ethics approval}

Not applicable.

\section{Consent for publication}

Not applicable.

\section{Competing interests}

The authors declare that they have no competing interests.

\section{Publisher's Note}

Springer Nature remains neutral with regard to jurisdictional claims in published maps and institutional affiliations.

\section{Author details}

${ }^{1}$ Department of Pathology, Microbiology, and Immunology, UC Davis, Davis, CA 95616, USA. ²Department of Entomology and Nematology, University of California, Davis, CA 95616, USA. ${ }^{3}$ Department of Biochemistry and Molecular Medicine, MIND Institute and UC-Davis Genome Center, University of California, Davis, CA 95616, USA.

Received: 15 December 2017 Accepted: 26 March 2018 Published online: 04 April 2018

\section{References}

1. WHO. Achieving the malaria MDG target. 2015. http://www.who.int/malaria/ publications/atoz/9789241509442/en/. Accessed 1 Feb 2018.

2. Ranson H, Lissenden N. Insecticide resistance in African Anopheles mosquitoes: a worsening situation that needs urgent action to maintain malaria control. Trends Parasitol. 2016;32:187-96.

3. Cisse MBM, Keita C, Dicko A, Dengela D, Coleman J, Lucas B, et al. Characterizing the insecticide resistance of Anopheles gambiae in Mali. Malar J. $2015 ; 14: 327$ 
4. Main BJ, Lee Y, Collier TC, Norris LC, Brisco K, Fofana A, et al. Complex genome evolution in Anopheles coluzzii associated with increased insecticide usage in Mali. Mol Ecol. 2015;24:5145-57.

5. Norris LC, Main BJ, Lee Y, Collier TC, Fofana A, Cornel AJ, et al. Adaptive introgression in an African malaria mosquito coincident with the increased usage of insecticide-treated bed nets. Proc Natl Acad Sci USA. 2015;112:815-20.

6. Lee Y, Marsden CD, Norris LC, Collier TC, Main BJ, Fofana A, et al. Spatiotemporal dynamics of gene flow and hybrid fitness between the $M$ and $\mathrm{S}$ forms of the malaria mosquito, Anopheles gambiae. Proc Natl Acad Sci USA. 2013;110:19854-9.

7. Ranson H, Abdallah H, Badolo A, Guelbeogo WM, Kerah-Hinzoumbé C,

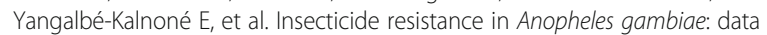
from the first year of a multi-country study highlight the extent of the problem. Malar J. 2009;8:299.

8. Ranson H, N'Guessan R, Lines J, Moiroux N, Nkuni Z, Corbel V. Pyrethroid resistance in African anopheline mosquitoes: what are the implications for malaria control? Trends Parasitol. 2011;27:91-8

9. Trape J-F, Tall A, Diagne N, Ndiath O, Ly AB, Faye J, et al. Malaria morbidity and pyrethroid resistance after the introduction of insecticide-treated bednets and artemisinin-based combination therapies: a longitudinal study. Lancet Infect Dis. 2011;11:925-32.

10. Hemingway J, Ranson $\mathrm{H}$. Insecticide resistance in insect vectors of human disease. Annu Rev Entomol. 2000;45:371-91.

11. Hemingway J. The molecular basis of two contrasting metabolic mechanisms of insecticide resistance. Insect Biochem Mol Biol. 2000;30:1009-15.

12. Müller P, Warr E, Stevenson BJ, Pignatelli PM, Morgan JC, Steven A, et al. Field-caught permethrin-resistant Anopheles gambiae overexpress CYP6P3, a P450 that metabolises pyrethroids. PLoS Genet. 2008;4:e1000286.

13. Stevenson BJ, Bibby J, Pignatelli P, Muangnoicharoen S, O'Neill PM, Lian L-Y, et al. Cytochrome P450 6M2 from the malaria vector Anopheles gambiae metabolizes pyrethroids: Sequential metabolism of deltamethrin revealed. Insect Biochem Mol Biol. 2011;41:492-502.

14. Müller P, Donnelly MJ, Ranson H. Transcription profiling of a recently colonised pyrethroid resistant Anopheles gambiae strain from Ghana. BMC Genomics. 2007:8:36

15. Tene BF, Poupardin R, Costantini C, Awono-Ambene P, Wondji CS, Ranson $H$, et al. Resistance to DDT in an urban setting: common mechanisms implicated in both $\mathrm{M}$ and $\mathrm{S}$ forms of Anopheles gambiae in the city of Yaoundé Cameroon. PLoS One. 2013;8:e61408.

16. Mulamba C, Irving $H$, Riveron JM, Mukwaya LG, Birungi J, Wondji CS. Contrasting Plasmodium infection rates and insecticide susceptibility profiles between the sympatric sibling species Anopheles parensis and Anopheles funestus s.s: a potential challenge for malaria vector control in Uganda. Parasit Vectors. 2014;7:71.

17. Nikou D, Ranson H, Hemingway J. An adult-specific CYP6 P450 gene is overexpressed in a pyrethroid-resistant strain of the malaria vector, Anopheles gambiae. Gene. 2003;318:91-102.

18. David J-P, Strode C, Vontas J, Nikou D, Vaughan A, Pignatelli PM, et al. The Anopheles gambiae detoxification chip: A highly specific microarray to study metabolic-based insecticide resistance in malaria vectors. Proc Natl Acad Sci USA. 2005;102:4080-4.

19. Awolola TS, Oduola OA, Strode C, Koekemoer LL, Brooke B, Ranson H. Evidence of multiple pyrethroid resistance mechanisms in the malaria vector Anopheles gambiae sensu stricto from Nigeria. Trans R Soc Trop Med Hyg. 2009;103:1139-45.

20. Djouaka RF, Bakare AA, Coulibaly ON. Expression of the cytochrome P450s, CYP6P3 and CYP6M2 are significantly elevated in multiple pyrethroid resistant populations of Anopheles gambiae s.s. from southern Benin and Nigeria. BMC Genomics. 2008;9:538.

21. McLaughlin LA, Niazi U, Bibby J, David J-P, Vontas J, Hemingway J, et al. Characterization of inhibitors and substrates of Anopheles gambiae CYP6Z2. Insect Mol Biol. 2008;17:125-35.

22. Corbel V, N'Guessan R, Brengues C, Chandre F, Djogbenou L, Martin T, et al. Multiple insecticide resistance mechanisms in Anopheles gambiae and Culex quinquefasciatus from Benin, West Africa. Acta Trop. 2007;101:207-16.

23. Namountougou M, Simard F, Baldet T, Diabaté A, Ouédraogo JB, Martin T, et al. Multiple insecticide resistance in Anopheles gambiae s.l. populations from Burkina Faso, West Africa. PLoS One. 2012;7:e48412.

24. Hardstone MC, Leichter CA, Scott JG. Multiplicative interaction between the two major mechanisms of permethrin resistance, $k d r$ and cytochrome P450monooxygenase detoxification, in mosquitoes. J Evol Biol. 2009;22:416-23.
25. Lanzaro GC, Touré YT, Carnahan J, Zheng L, Dolo G, Traoré S, et al. Complexities in the genetic structure of Anopheles gambiae populations in west Africa as revealed by microsatellite DNA analysis. Proc Natl Acad Sci USA. 1998:95:14260-5.

26. Schmidt JM, Good RT, Appleton B, Sherrard J, Raymant GC, Bogwitz MR, et al. Copy number variation and transposable elements feature in recent, ongoing adaptation at the Cyp6g1 locus. PLoS Genet. 2010;6:e1000998.

27. Johnson BR, Atallah J, Plachetzki DC. The importance of tissue specificity for RNA-seq: highlighting the errors of composite structure extractions. BMC Genomics. 2013;14:586.

28. Ingham VA, Jones CM, Pignatelli P, Balabanidou V, Vontas J, Wagstaff SC, et al. Dissecting the organ specificity of insecticide resistance candidate genes in Anopheles gambiae: known and novel candidate genes. BMC Genomics. 2014; 15:1018.

29. Vontas J, Blass C, Koutsos AC, David J-P, Kafatos FC, Louis C, et al. Gene expression in insecticide resistant and susceptible Anopheles gambiae strains constitutively or after insecticide exposure. Insect Mol Biol. 2005: 14:509-21.

30. Brogdon W, Chan A. Guidelines for evaluating insecticide resistance in vectors using the CDC bottle bioassay/methods in Anopheles research. CDC Atlanta: CDC Technical Report; 2010. p. 343.

31. WHO. Test procedures for insecticide resistance monitoring in malaria vector mosquitoes. 2016. http://www.who.int/malaria/publications/atoz/ 9789241511575/en/. Accessed 1 Feb 2018.

32. Bolger AM, Lohse M, Usadel B. Trimmomatic: a flexible trimmer for Illumina sequence data. Bioinformatics. 2014;30:2114-20.

33. Dobin A, Davis CA, Schlesinger F, Drenkow J, Zaleski C, Jha S, et al. STAR: ultrafast universal RNA-seq aligner. Bioinformatics. 2013;29:15-21.

34. Robinson MD, McCarthy DJ, Smyth GK. edgeR: a Bioconductor package for differential expression analysis of digital gene expression data. Bioinformatics. 2010;26:139-40.

35. Law CW, Chen Y, Shi W, Smyth GK. voom: precision weights unlock linear model analysis tools for RNA-seq read counts. Genome Biol. 2014:15:R29.

36. Ritchie ME, Phipson B, Wu D, Hu Y, Law CW, Shi W, et al. limma powers differential expression analyses for RNA-sequencing and microarray studies. Nucleic Acids Res. 2015;43:e47.

37. Smyth GK. Linear models and empirical Bayes methods for assessing differential expression in microarray experiments. Stat Appl Genet Mol Biol. 2004;3:3.

38. Layer RM, Chiang C, Quinlan AR, Hall IM. LUMPY: a probabilistic framework for structural variant discovery. Genome Biol. 2014;15:R84.

39. Reimand J, Arak T, Adler P, Kolberg L, Reisberg S, Peterson H, et al. g:Profiler - a web server for functional interpretation of gene lists (2016 update). Nucleic Acids Res. 2016:44:W83-9.

40. Gonzalez FJ. Role of cytochromes P450 in chemical toxicity and oxidative stress: studies with CYP2E1. Mutat Res. 2005:569:101-10.

41. Müller P, Chouaïbou M, Pignatelli P, Etang J, Walker ED, Donnelly MJ, et al. Pyrethroid tolerance is associated with elevated expression of antioxidants and agricultural practice in Anopheles arabiensis sampled from an area of cotton fields in northern Cameroon. Mol Ecol. 2008;17:1145-55.

42. Chiu T-L, Wen Z, Rupasinghe SG, Schuler MA. Comparative molecular modeling of Anopheles gambiae CYP6Z1, a mosquito P450 capable of metabolizing DDT. Proc Natl Acad Sci USA. 2008;105:8855-60.

43. Ibrahim SS, Ndula M, Riveron JM, Irving H, Wondji CS. The P450 CYP6Z1 confers carbamate/pyrethroid cross-resistance in a major African malaria vector beside a novel carbamate-insensitive N485I acetylcholinesterase-1 mutation. Mol Ecol. 2016:25:3436-52

44. Irving H, Riveron JM, Ibrahim SS, Lobo NF, Wondji CS. Positional cloning of rp2 QTL associates the P450 genes CYP6Z1, CYP6Z3 and CYP6M7 with pyrethroid resistance in the malaria vector Anopheles funestus. Heredity. 2012:109:383-92. 